\title{
AVALIAÇÃO DA EFICIÊNCIA DA MISTURA PRONTA DE GLYPHOSATE + 2,4-D NO CONTROLE DA Commelina virginica L. EM CITROS ${ }^{\mathbf{1}}$
}

\author{
HAMILTON H. RAMOS ${ }^{2}$, JULIO C. DURIGAN ${ }^{3}$
}

\section{RESUMO}

Um dos herbicidas mais utilizados para o controle de plantas daninhas em pós-emergência nos pomares cítricos paulistas é o glyphosate. No entanto, este herbicida aplicado isol adamente e nas doses recomendadas, tem proporcionado seleção da planta daninha trapœraba (Conunelina nelina virginica L.), devido à grande tolerância da mesma, somada à eficiente eliminação das demais espécies da comunidade infestante. Em vista disso, e pela falta de opções, faz-se necessária a pesquisa de outros herbicidas ou misturas de herbicidas que sejam eficientes no controle da trapoeraba, de baixa toxicidade para os aplicadores, sistêmicos, e que sejam seletivos às plantas cítricas. O presente trabalho foi de senvolvido com o objetivo de estudar a eficiência da mistura pronta dos herbicidas glyphosate $+2,4-\mathrm{D}$ amina no controle da trapoeraba em citros [Citrus sinensis (L.) Osbeck], em comparação com estes mesmos herbicidas aplicados isoladamente, bem como os possíveis ef ei to s fit ot óx ic os da me sma à cu lt ura. O experimento foi instalado na região de Catanduva - SP, em um pomar de laranja 'Pera', clone Rio, enxertada sobre limão "Cravo", com dez anos de idade, plantado em um espaçamento de 8,0 x 6,5 m. A aplicação dos herbicidas foi realizada em 20 de fevereiro de 1991, de forma dirigida, em pósemergência tardia da trapoeraba, quando a mesma já flor escia e tinha altu ra variável entre 15 e 60 $\mathrm{cm}$. A análise e interpretação dos resultados obtidos mostraram que a mistura pronta de glyphosate $+2,4-\mathrm{D}$ apresenta um controle superior da trapoeraba em relação aos produtos aplicados isoladamente, não havendo diferenças significativas no controle para doses superiores a $0,60+0,80 \mathrm{~kg}$ i.a./ha. Os dados obtidos reforçam a teoria de sinergismo entre os dois produtos. Em nenhum dos tratamentos foi verificado sintomas visuais de intoxicação nas laranjeiras.

Palavras chave: Citrus sinensis, trapoeraba, controle químico

\section{ABSTRACT \\ Efficacy evaluation of ready mixture of glyphosate + 2,4-D in Commelina virginica $\mathbf{L}$. control in citrus}

In citrus orchard of Sao Paulo state, Brazil, one of the most employed post-emergency herbicide for weed control is glyphosate. Howe ver, when this herbicide is applied alone and at the recommended rates, it has been observed a selection of the weed Commelina virginica L. due to its great tolerance and the elimination of other weeds. Because of that, other chemicals must be studied to solve the problem. In this particular case, the herbicide should be sistemic and have a low toxicity to the operator and to the citrus trees. This work was conducted to study the efficacy of a ready mixture of glyphosate +2,4-D compared to the products

\footnotetext{
${ }^{1}$ Parte da dissertação apresentada à Faculdade de Ciencias Agrárias e Veterinárias - UNESP. Campus de Jaboticabal. Recebido para publicação em 21/06/95 e a forma revisada em 16/02/96.

${ }^{2}$ Pesquisador Científico, Seção de Máquinas Implantadoras de Culturas e Aplicadoras de Defensivos, Instituto Agronômico de Campinas (IAC), Caixa Postal 26, CEP 13.201-970, Jundiaí, SP.

${ }^{3}$ Departamento de Defesa Fitossanitária, FCVA - UNESP, Campus de Jaboticabal.
} 
sprayed alone in orange orchards [Citrus sinensis (L.) Osbeck]. The phytotoxicity effects were studied too. The experiment was carried out in Catanduva, São Paulo, Brazil, in a ten years old orange orchard, with $8,0 \times 6,0 \mathrm{~m}$ spacing. On february, $20^{\mathrm{h}}$, 1991, the herbicides were sprayed directly on $15-60 \mathrm{~cm}$ height Commelina during its flowering. The results showed that the ready

\section{INTRODUÇÃO}

O Brasil se destaca como um dos principais países produtores de citros e o maior exportador de suco concentrado congel ado. O Estado de São Paulo é o principal produtor de citros do país, sendo responsável por aproximadamente $80 \%$ da produção total, cabendo $20 \%$ aos Estados da Bahia, Minas Gerais, Rio de Janeiro, Rio Grande do Sul, Sergipe e outros. Em 980.000 hectares do solo brasileiro (IBGE, 1992), estão cerca de 245 milhões de plantas. Entretanto, enquanto países como o Japão e Israel produzem, em média, 40 a 60 t/ha e outros como USA, Itália e Espanha têm produção da ordem de 30 a 40 t/ha, no Brasil o rendimento médio não chega a $20 \mathrm{t} / \mathrm{ha}$ (De Negri, 1988).

O método de controle químico de plantas daninhas nos pomares cítricos é uma prática bastante utilizada em várias regiões do mundo, podendo-se citar como exemplo os EUA que já em 1975 tratavam cerca de 50\% de sua área total (Lange et al., 1975), enquanto que no Brasil tal prática é ainda pouco expressiva devido a escassez de conhecimentos sobre a eficácia dos produtos químicos registrados e existentes no mercado, formas de aplicação, limitações de uso e cuidados na aplicação. Além disso, há grande escassez de pesquisas básicas com relação aos efeitos do uso contínuo de herbicidas residuais no pomar e dos principais períodos de interferência das plantas daninhas (Durigan, 1988).

A aplicação por vários anos consecutivos, de um mesmo herbicida, pode levar à seleção de algumas espécies de plantas daninhas, aumentando sensivelmente a sua importância na área. $\mathrm{O}$ herbicida glyphosate por exemplo, mixture was better in Commelina control than other treatments. No significant differences were observed for the rates greater than $0,60+0,80 \mathrm{~kg}$ a.i./ha. The results reinforced the sinergism theory within both products. None of the treatments caused visual phytotoxicity to orange trees.

Key words: Commelina sp, Cilrus sinensis, chemical control

aplicado isoladamente e nas doses recomendadas, tem proporcionado se leção da trapoe raba (Commelina virginica L.), de vido à grande tole rância da mesma (Durigan et al., 1988; Lorenzi, 1986), somada à eficiente eliminação das demais espécies da comunidade infestante.

A trapoeraba (Commelina virginica L.) é uma planta daninha perene, herbácea, ereta ou semi-prostrada, muito ramificada, com caules articulados grossos e alongados, medindo de 30 a $70 \mathrm{~cm}$ de altura, com reprodução por sementes e através de curtos rizomas. Esta planta daninha é bastante frequente, infestando lavouras anuais e perenes, hortas, margens de canais e terren os baldios. Adapta-se muito bem em solos argilosos, úmidos 'e sombreados (Lorenzi, 1982). É uma infestante bastante comum nos pomares cítricos do Estado de São Paulo devido ao seu difícil controle químico, principalmente no estádio adulto. Um dos herbicidas mais utilizados para o controle de plantas daninhas em pós-emergência nos pomares cítricos paulistas é o glyphosate, que, no entanto, tem baixa eficiência sobre esta planta daninha (Durigan, 1988; Lorenzi, 1986), fazendo com que outro herbicida seja utilizado posteriormente para o controle da mesma. O herbicida mais utilizado para esta finalidade é o paraquat que, por ser altamente tóxico, pode se constituir em um sério problema para pequenos e médios citricult ore s que não possuam mão-deobra treinada para sua manipulação e aplicação. Al ém desse fato, a portaria $n^{\circ} 329$ de 2 de setembro de 1985 estabelece em seu artigo $2^{\circ}$ que este herbicida pode ser comercializado somente através de venda aplicada, o que dificultou em muito sua aquisição por parte dos citricultores. Cardi (1980), citado por Hammerton (1981),

Planta Daninha, v. 14, n. 1, 1996. 
constatou que esta planta daninha mostrou-se resistente ao paraquat. No entanto, Hammerton (1981), explica que is to pode ter acontecido devido a ineficiência da pulverização em atingir as folhas mais internas das plantas. Em vista disso, e pela falta de opções, faz-se necessária a pesquisa de outros herbicidas que sejam eficazes no controle da trapoeraba, menos tóxicos para os aplicadores, sistêmicos, e que causem o mínimo possível de fitotoxicidade às plantas cítricas.

$\mathrm{Na}$ tentativa de aumentar a eficácia do glyphosate, Galli (1991) estudou a eficiência de diversos produtos misturados a este herbicida, no controle de Commelina virginica L. Neste trabalho foi verificado que os melhores níveis de controle foram obtidos com a mistura pronta de glyphosate $+2,4-\mathrm{D}$, em todas as avaliações, alcançando, aos 28 dias após a aplicação, um controle médio de 95 a $98 \%$, para as doses de 1,68 e 2,24 kg e.a./ha, respectivamente. Nos demais tratamentos, envolvendo misturas de glyphosate, observou um efeito visual mais rápido, principalmente com diurona (14 D.A.A.), porém, es sa te ndên cia não se verificou na última avaliação (54 D.A.A.), quando o glyphosate isoladamente mostrou-se semelhante às misturas com KCI $(0,5 \% \mathrm{p} / \mathrm{v})$ ou uréia $(0,5 \% \mathrm{p} / \mathrm{v})$ e superior à diurona.

Tollervey et al. (1979) constataram, na cultura dos citros que, dos herbicidas testados, a mis tura glyphosate a $5,0 \mathrm{~kg} / \mathrm{ha}+2,4-\mathrm{D}$ a 2,0 $\mathrm{kg} / \mathrm{ha}$, apresentou um controle visivelmente melhor das plantas daninhas até quatorze semanas após a apl icação. O controle com glyphosate isolado foi superior por dez semanas após a apl icação, mas decresceu de 50\% para $8 \%$ na décima quarta semana. Eles concluiram pela possibilidade de um efeito sinergístico entre $o$ glyphosate e o 2,4-D, que ajudaria a explicar esta divergência. Este efeito sinergístico também foi notado por Suwunnamek \& Parker (1975), para o controle de Cyperus rotundus L. e por Moshier (1980) e Flint \& Barret (1989) para Convolvulus arvensis $\mathrm{L}$.

No entanto, Carvalho et al. (1991 a) estudaram a eficiência da mistura pronta de gl yp ho sa te + 2,4 -D, em co mp ar ação com glyphosate e 2,4-D aplicados isoladamente, no controle de plantas daninhas da cultura do cacau e verificaram que nenhum dos tratamentos testados apresentou controle satisfatório para trapoeraba (Commelina virginica L.), capim-amargoso [Digitaria insularis (L.) Mez ex Ekman] e falsaserralha (Emilia sonchifolia DC.).

No presente trabalho, estudou-se a eficiência da mistura pronta de glyphosate + 2,4-D no controle da planta daninha trapoeraba na cultura do citros, bem como os possíveis efeitos fitotóxicos da mesma à cultura.

\section{MATERIAL E MÉTODOS}

O experimento foi instalado emum pomar de laranja 'Pera', clone Rio, enxertada sobre limão 'Cravo', com dez anos de idade, plantado em um espaçamento de $8,0 \times 6,5 \mathrm{~m}$ e pertencente ao pomar comercial da Estância Sta. Angela, em Catanduva - SP, localizada a $21^{\circ} 08^{\prime} 16^{\prime \prime}$ de latitude sul e $48^{\circ} 58^{\prime} 22^{\prime \prime}$ de longitude WGr, a uma altitude de $503 \mathrm{~m}$. O clima da região, segundo a Clas sificação de Köppen, é o Cwa, subtropical com inverno relativamente seco. A precipitação média anual é de $1.500 \mathrm{~mm}$ e a do mês mais seco é de $25,0 \mathrm{~mm}$. A temperatura média anual é de aproximadamente $22^{\circ} \mathrm{C}$ e a do mês mais frio é inferior a $16^{\circ} \mathrm{C}$.

O solo da área onde foi instalado o experimento foi classificado, pela Assessoria de Conservação do Solo e Água da VIII Região Administrativa, Dira de São José do Rio Preto, como Podzólico Vermel ho - Amarelo, textura arenosa média, com 'A' abrupto. Apresentava uma topografia relativamente plana e era bem drenado.

O delineamento experimental utilizado foi o de blocos ao acaso, com onze tratamentos e quatro repetições. Utilizaram-se parcelas de 4,0 x $6,5 \mathrm{~m}$, perfazendo uma área total de $26,0 \mathrm{~m}^{2}$, que corre spondia a uma planta de citros. A aplicação dos herbicidas foi realizada em duas faixas $(2,0 \mathrm{~m})$ laterais às linhas da cultura, send o uma de cada lado da planta, deixando-se uma faixa de $0,5 \mathrm{~m}$ 
entre as parcelas corno bordadura.

Os herbicidas testados foram a mistura pronta de glyphosate + 2,4-D amina $(161,9+$ $202,7 \mathrm{~g}$ do ingrediente ativo/l), 2,4-D amina (868g do ingrediente ativo/l) e glyphosate $(480 \mathrm{~g}$ do ingrediente ativo/l) e os tratamentos utilizados estão descritos no Tabela 1. A aplicação dos herbicidas foi realizada em 20 de fevereiro de 1991, de forma dirigida, em pós-emergência tardia da trapoeraba, quando a mesma se encontrava em flores cimento, túrgida, com altura variável entre 15 e $60 \mathrm{~cm}$ e ocupando $100 \%$ da área das parcelas. Utilizou-se um pulverizador costal, à pressão constante (mantida por $\mathrm{CO}_{2}$ comprimido) de 45 $\mathrm{lbf} / \mathrm{pol}^{\mathrm{i}}\left(3,16 \mathrm{~kg} / \mathrm{cm}^{2}\right)$, equipado com barra de $2,0 \mathrm{~m}$ de largura e quatro bicos* de jato plano (tipo leque), com consumo equivalente a 400 I de calda por ha.

Por ocasião da aplicação dos produtos químicos, a temperatura ambiente era de $28^{\circ} \mathrm{C}$ à sombra e a te mperat ura do solo, a $5 \mathrm{~cm}$ de prof undidade e sob a "copa" das plantas, era de $25^{\circ} \mathrm{C}$. A insolação era alta, o solo estava úmido na superficie e a velocidade do vento variou entre $1 \mathrm{e}$ $3 \mathrm{~km} / \mathrm{h}$. A aplicação iniciou-se às 13:20 hs e estendeu-se até às 14:15 horas.

Foram realizadas avaliações visuais de controle da trapoeraba e sintomas de fitotoxicidade nas plantas de laranja aos 12, 20, 29, 42, 63 e 91 dias após a aplicação (D.A.A.). Aos 29 e 98 dias após a aplicação dos herbicidas, realizou-se a coleta de plantas daninhas para determinação do peso da matéria seca.

TABELA 1: Tratamentos utilizados para o controle da trapoeraba (Commelina virginica L.), com suas respectivas doses do ingre diente ativo e do produto comercial por ha. Catanduva - SP, 1991.

\begin{tabular}{|c|c|c|c|}
\hline \multirow[t]{2}{*}{$\mathrm{N}^{\circ}$} & \multirow[t]{2}{*}{ TRATAMENTOS } & \multicolumn{2}{|c|}{ DOSES } \\
\hline & & (kg i.a./ha) & (I p.c./ha) \\
\hline 01 & glyphosate $+2,4-\mathrm{D}^{(\mathrm{a})}$ & $0,65+0,81$ & 4,00 \\
\hline 02 & glyphosate $+2,4-\mathrm{D}$ & $0,81+1,01$ & 5,00 \\
\hline 03 & glyphosate $+2,4-\mathrm{D}$ & $0,97+1,22$ & 6,00 \\
\hline 04 & glyphosate $+2,4-\mathrm{D}$ & $1,13+1,42$ & 7,00 \\
\hline 05 & glyphosate $+2,4-\mathrm{D}$ & $1,30+1,62$ & 8,00 \\
\hline 06 & $2,4-\mathrm{D}^{(\mathrm{b})}$ & 0,77 & 0,89 \\
\hline 07 & $2,4-\mathrm{D}$ & 1,54 & 1,78 \\
\hline 08 & glyphosate $^{(c)}$ & 0,65 & 1,35 \\
\hline 09 & glyphosate & 1,30 & 2,70 \\
\hline 10 & Testemunha mantida no limpo & - & - \\
\hline 11 & Testemunha mantida infestada & - & - \\
\hline
\end{tabular}

(a) Command (b) U 46 D Fluid 2,4-D (c) Roundup SAqC

Para as avaliações visuais de controle das plantas daninhas e de sintomas de fitotoxicidade na cul tura, utilizou-se das escalas de notas da Alam (1974) e da EWRC (1964), respectivamente.

* Albuz vermelho
Na escala da Alam (1974), a nota 01 representa 0 a $40 \%$ de controle, a nota 02 de 41 a $60 \%$, a nota 03 de 61 a $70 \%$, a nota 04 de 71 a $80 \%$, a nota 05 de 81 a $90 \%$ e a nota 06 , de 91 a $100 \%$ de controle. Para o EW RC (1964), a nota 01 corresponde à ausência de sintomas de 
fitotoxicidade e 09, à morte das plantas.

Para a determinação do peso da matéria seca, coletou-se toda a parte aérea das plantas de trapoeraba contidas em $0,4 \mathrm{~m}^{2}$ da parcela, as quais foram pesadas e acondicionad as em sacos de papel devidamente identificados. Posteriormente foram levadas à estufa de circulação e renovação forçada de ar a $60-70^{\circ} \mathrm{C}$ até peso constante, após o que, todo o material foi pesado.

\section{RESULTADOS E DISCUSSÃO}

As médias das notas de controle atribuídas nas avaliações visuais, em cada uma das épocas de avaliação, são mostradas no Tabela 2 .

$\mathrm{Na}$ Tabela 3 são apresentados os resultados da análise estatística, referentes às notas atribuídas nas avaliações visuais de controle. A análise também contempla os efeitos interativos entre os herbicidas e as épocas de avaliação. Verifica-se que os herbicidas apresentam efeitos diferentes para o controle da trapoeraba, ao nível de $1 \%$ de probabilidade, pelo teste F. Observa-se também haver influência das épocas de avaliação e da interação herbicidas $x$ épocas com significân cia a nível de $1 \%$ de probabilidade, indicando existir uma dependência entre os efeitos destes fatores.

Aplicando-se o teste de Tukey ao nível de $5 \%$ de probabilidade para a interação herbicidas $\mathrm{x}$ épocas de avaliação (Tabela 4), observa-se que os melhores controles for am conseguidos com a mistura pronta de glyphosate $+2,4-\mathrm{D}$ amina nas doses de $1,30+1,62 ; 1,13+1,42$ e $0,97+1,22 \mathrm{~kg}$ do ingrediente ativo/ha, os quais já possibilitavam porcentagens de controle da trapoeraba acima de $70 \%$, ao s 12 D.A.A. (dias após a aplicação), evoluindo para mais de $80 \%$ a part ir dos 29 D.A.A.. O controle obtido nestes tratamentos foi igualado pelo obtido com a mes ma mistura na dose de $0,81+1,01 \mathrm{~kg}$ do ingredie nte ativo/ha a partir de 29 D.A.A. e pelas doses de $0,65+0,81 \mathrm{~kg}$ do ingrediente ativo/ha a partir de 42 D.A.A.

TABELA 2. Valores médios ${ }^{(1)}$ das notas atribuídas nas avaliações visuais de controle da trapoeraba (Commelina virginica L.), em cada uma das épocas de avaliação. Catanduva - SP, 1991.

\begin{tabular}{|c|c|c|c|c|c|c|}
\hline \multirow[t]{2}{*}{ TRATAMENTOS } & \multicolumn{6}{|c|}{ ÉPOCAS DE AVALIAÇÃO (D.A.A.) } \\
\hline & 12 & 20 & 29 & 42 & 63 & 91 \\
\hline \multicolumn{7}{|l|}{ glyphosate $+2,4-\mathrm{D}^{(2)}$} \\
\hline $0,65+0,81$ & 3,5 & 3,6 & 4,4 & 5,3 & 5,5 & 5,5 \\
\hline $0,81+1,01$ & 3,8 & 4,0 & 5,3 & 5,8 & 5,8 & 5,9 \\
\hline $0,97+1,22$ & 4,1 & 4,9 & 5,3 & 5,5 & 5,8 & 5,9 \\
\hline $1,13+1,42$ & 4,8 & 4,8 & 5,6 & 5,9 & 5,8 & 5,8 \\
\hline $1,30+1,62$ & 4,9 & 5,0 & 5,6 & 5,8 & 6,0 & 6,0 \\
\hline \multicolumn{7}{|l|}{$2,4-\mathrm{D}^{(2)}$} \\
\hline 0,77 & 1,1 & 1,5 & 2,0 & 2,4 & 1,4 & 1,3 \\
\hline 1,54 & 1,1 & 2,0 & 2,3 & 3,1 & 2,5 & 1,6 \\
\hline \multicolumn{7}{|l|}{ glyphosate $^{(2)}$} \\
\hline 0,65 & 2,5 & 2,3 & 3,0 & 4,4 & 5,0 & 5,4 \\
\hline 1,30 & 2,9 & 2,9 & 3,8 & 4,9 & 5,8 & 5,6 \\
\hline testemunha mantida infestada & 1,0 & 1,0 & 1,0 & 1,0 & 1,0 & 1,0 \\
\hline \multicolumn{7}{|l|}{ (1) Escala da ALAM (1974) } \\
\hline (2) Doses em kg i.a./ha & & & & & & \\
\hline D.A.A. - Dias Após a Aplicação & & & & & & \\
\hline
\end{tabular}

Planta Daninha, v. 14, n. 1, 1996. 
As avaliações realizadas até 42 D.A.A. evidenciam melhor efeito da mistura em relação aos produtos isolados no controle de $C$. virginica, estando estes dados em conformidade com Durigan et al. (1988), Galli (1991) e Carvalho et al. (1991b), e colaboram com a hipótese de um possível sinergismo entre o glyphosate e o 2,4-D, tal como foi sugerido por Tollervey et al. (1979), Suwunnamek \& Parker (1975) e Flint \& Barret (1989).

TABELA 3: Análise de variância das avaliações visuais de controle da trapoeraba (Commelina virginica L.) para os vários herbicidas testados, nas diversas épocas de avaliação. Catanduva - SP, 1991.

\begin{tabular}{lcrrr}
\hline C. VARIAÇ̃̃O & G.L. & \multicolumn{1}{c}{ S.Q. } & \multicolumn{1}{c}{ Q.M. } & \multicolumn{1}{c}{ F } \\
\hline BLOCOS & 3 & 3,5787 & 1,1929 & $3,69^{*}$ \\
HERBICIDAS (H) & 8 & 398,4583 & 40,8073 & $154,15^{* *}$ \\
RESÍDUO (A) & 24 & 7,7546 & 0,3231 & \\
\hline (PARCELAS) & 35 & 409,7916 & & $166,01^{* *}$ \\
\hline ÉPOCAS (E) & 5 & 95,5556 & 19,1111 & $9,24^{* *}$ \\
INTERAÇÃO H X E & 40 & 42,5694 & 1,0642 & \\
RESÍDUO (B) & 135 & 15,5417 & 0,1151 & \\
\hline TOTAL & 215 & 563,4583 & & \\
\hline
\end{tabular}

Coeficiente de variação para herbicidas: 13,6

Coeficiente de variação para épocas: $\quad 8,1$

TABELA 4: Teste de Tukey para a interação herbicidas $x$ épocas de avaliação do controle da trapoeraba (Commelina virginica L.). Catanduva - SP, 1991.

\begin{tabular}{|c|c|c|c|c|c|c|}
\hline \multirow{3}{*}{$\begin{array}{c}\text { HERBICIDAS } \\
\text { (kg i.a./ha) }\end{array}$} & \multicolumn{6}{|c|}{ ÉPOCAS DE AVALIAÇ̃̈O (D.A.A.) } \\
\hline & 12 & 20 & 29 & 42 & 63 & 91 \\
\hline & \multicolumn{6}{|c|}{ MEDIAS DAS NOTAS DE CONTROLE $\overline{\mathbf{E}}^{7 \pi}$} \\
\hline glyphosate $+2,4-\mathrm{D}(1,30+1,62)$ & $4,9 \mathrm{c} \mathrm{A}$ & 5,0 bc $\mathrm{A}$ & 5,6 ab $\mathrm{A}$ & 5,8 a $\mathrm{A}$ & 6,0 a $A$ & 6,0 a $\mathrm{A}$ \\
\hline glyphosate $+2,4-\mathrm{D}(1,13+1,42)$ & $4,8 \mathrm{~b} \mathrm{~A}$ & $4,8 \mathrm{~b} \mathrm{AB}$ & 5,6 a A & 5,9 a $A$ & 5,8 a $\mathrm{AB}$ & 5,8 a $\mathrm{A}$ \\
\hline glyphosate $+2,4-\mathrm{D}(0,97+1,22)$ & $4,1 \mathrm{c} \mathrm{AB}$ & 4,9 b A & $5,3 \mathrm{ab} \mathrm{A}$ & $5,5 \mathrm{ab} \mathrm{AB}$ & 5,8 a $\mathrm{AB}$ & 5,9 a $\mathrm{A}$ \\
\hline glyphosate $+2,4-\mathrm{D}(0,81+1,01)$ & 3,8 b B & $4,0 \mathrm{~b} \mathrm{BC}$ & 5,3 a $\mathrm{A}$ & 5,8 a $\mathrm{A}$ & 5,8 a $\mathrm{AB}$ & 5,9 a $\mathrm{A}$ \\
\hline glyphosate $+2,4-\mathrm{D}(0,65+0,81)$ & $3,5 \mathrm{c} \mathrm{BC}$ & $3,6 \mathrm{c} \mathrm{CD}$ & 4,4 b B & 5,3 a $\mathrm{AB}$ & 5,5 a $\mathrm{AB}$ & 5,5 a $A$ \\
\hline glyphosate $(1,30)$ & $2,9 \mathrm{~d} \mathrm{CD}$ & $2,9 \mathrm{~d} \mathrm{DE}$ & $3,8 \mathrm{c} \mathrm{BC}$ & 4,9 b BC & 5,8 a $\mathrm{AB}$ & 5,6 a A \\
\hline glyphosate $(0,65)$ & $2,5 \mathrm{~cd} \mathrm{D}$ & $2,3 \mathrm{~d} \mathrm{EF}$ & $3,0 \mathrm{c} \mathrm{CD}$ & $4,4 \mathrm{~b} \mathrm{C}$ & 5,0 ab B & 5,4 a $A$ \\
\hline $2,4-\mathrm{D}(1,54)$ & $1,1 \mathrm{dE}$ & $2,0 \mathrm{bc} \mathrm{F}$ & 2,3 bc DE & 3,1 a D & $2,5 \mathrm{abC}$ & $1,6 \mathrm{~cd} \mathrm{~B}$ \\
\hline $2,4-\mathrm{D}(0,77)$ & $1,1 \mathrm{cE}$ & $1,5 \mathrm{bc} \mathrm{F}$ & $2,0 \mathrm{ab} \mathrm{E}$ & 2,4 a D & $1,4 \mathrm{bc} D$ & $1,3 \mathrm{c} \mathrm{B}$ \\
\hline
\end{tabular}

D.M.S. $($ TUKEY) $(H$ d. E) $=0.9 \quad$ D.M.S. $($ TUKEY $)(E \mathrm{~d}$. H $)=0,7$

Médias seguidas por uma mesma letra maiúscula não diferem entre si, numa mesma coluna, pelo teste de Tukey Médias seguidas por uma mesma letra minúscula não diferem entre si, numa mesma linha, pelo teste de Tukey D.A.A. - Dias Após a Aplicação $\quad$ (1) Escala da ALAM (1974)

A ação direta do glyphosate proporcionou controle muito baixo nos primeiros 20 D.A.A. No entanto, pode-se constatar que as pequenas lesões provocadas por este herbicida, auxiliaram a 
abreviar o ciclo da planta da ninha, talvez complementada pela ação de algum ti po de patógeno. A senescência da trapoeraba foi acelerada pela ação do glyphosate, fato que não foi constatado onde aplicou-se 2,4-D amina isoladamente.

Verifica-se também, pela análise da Tabela 4, que em todos os tratamentos onde se utilizou a mistura ou o glyphosate isolado, o controle tendeu a crescer até um máximo, que foi então mantido até o final das avaliações. Com a mistura, em todas as doses testadas, exceto na de $0,65+0,81 \mathrm{~kg}$ do ingredie nte ativo/lia, o controle da trapoeraba evoluiu até 29 D.A.A., quando ating iu o seu ápice, não declinando mais a partir de então até 91 D.A.A. Já os tratamentos com 2,4-D amina isolado, não mostraram controles que apresentassem médias de notas superiores a 3,1 mesmo com a maior dose $(1,54 \mathrm{~kg}$ do ingrediente ativo/ha).

Os tratamentos afetaram de forma estatisticamente diferente a matéria seca da trapoeraba, ao nível de $1 \%$ de probabilidade, pelo tes te $\mathrm{F}$ aos 29 D.A.A. Apl icando-se o teste de Tukey às médias (Tabela 5), verifica-se que os melhores controles foram obtidos com a mistura nas diferentes doses e os piores com 2,4-D amina e o gl yphosate isolados. Es tes resultados confirmam os obtidos nas avaliações visuais de controle aos 29 D.A.A.

A análise de variância realizada para a matéria seca da trapoeraba aos 98 D.A.A. (Tabela $6)$, permite observar que os tratamentos apresentam efeitos diferentes sobre a matéria seca da trapoeraba, ao nível de $1 \%$ de probabilidade, pelo teste $\mathrm{F}$, resultados estes semelhantes aos 29 D.A.A. Aplicando-se o teste de Tukey às médias de matéria seca da trapoeraba, nesta mesma época de coleta, constata-se que os melhores controles eram mantidos pela mistura e os piores pelo 2,4-D amina isolado. Estes resultados também confirmam os obtidos nas avaliações visuais de controle aos 91 D.A.A.

TABELA 5: Teste de Tukey aplicado ao peso médio de matéria seca da trapoeraba (Commelina virginica L.), aos 29 D.A.A. dos tratamentos. Catanduva - SP, 1991.

\begin{tabular}{lcl}
\hline TRATAMENTOS $^{(1)}$ & PESO MÉDIO DA MATÉRIA SECA $(\mathrm{g})^{(2)}$ \\
\hline Testemunha & 23,0 & $\mathrm{~A}$ \\
2,4-D $(0,77)$ & 19,6 & $\mathrm{AB}$ \\
2,4-D $(1,54)$ & 19,4 & $\mathrm{AB}$ \\
glyphosate $(1,30)$ & 15,2 & $\mathrm{ABC}$ \\
glyphosate $(0,65)$ & 14,3 & $\mathrm{ABC}$ \\
glyphosate $+2,4-\mathrm{D}(0,65+0,81)$ & 11,9 & $\mathrm{BC}$ \\
glyphosate $+2,4-\mathrm{D}(0,81+1.01)$ & 9,2 & $\mathrm{C}$ \\
glyphosate $+2,4-\mathrm{D}(1,30+1,62)$ & 8,1 & $\mathrm{C}$ \\
glyphosate $+2,4-\mathrm{D}(0,97+1,22)$ & 7,6 & $\mathrm{C}$ \\
glyphosate $+2,4-\mathrm{D}(1,13+1,42)$ & 7,1 & $\mathrm{C}$ \\
\hline F trat & 9,94 & $* *$ \\
C.V.(\%) & 26.7 & \\
D.M.S. (Tukey) a 5\% & 8,8 & \\
\hline
\end{tabular}

(1) Dosagens em kg i.a./ha

(2) Dados transformados $\mathrm{em} \sqrt{x+1}$

D.A.A. - Dias Após a Aplicação 
Portanto, pode-se conclu ir que, nas condições do experimento, a mistura pronta de glyphosate + 2,4-D amina apresenta um controle superior da trapoeraba (Commelina virginica L.), em relação a estes produtos aplicados is ol ad amente, sendo que não se observam diferenças no controle para doses superiores a $0,65+0,81 \mathrm{~kg}$ i.a. $/ \mathrm{ha}$.

Em nenhuma das avaliações realizadas se observaram quaisquer sintomasde fitotoxicidade nas laranjeiras, confirmando as observações de Cruz et al. (1986), Durigan et al. (1988) e Carvalho et al. (1991b), podendo-se concluir portanto, que para as condições do experimento, a mistura pronta de glyphosate +2 ,4-D amina, aplicada de forma dirigida em um pomar cítrico, em doses de até $1,30+1,62 \mathrm{~kg}$ do ingrediente ativo/ha, não causa fitotoxicidade às plantas.

TABELA 6: Teste de Tukey aplicado ao peso médio de matéria seca da trapoeraba (Commelina virginica L.) aos 98 D.A.A. dos tratamentos. Catanduva - SP, 1991.

\begin{tabular}{lrl}
\hline TRATAMENTOS $^{(1)}$ & PESO MÉDIO DA MATÉRIA SECA $(\mathrm{g})^{(2)}$ \\
\hline Testemunha & 12,8 & $\mathrm{~A}$ \\
2,4-D $(0,77)$ & 11,9 & $\mathrm{~A}$ \\
2,4-D $(1,54)$ & 11,4 & $\mathrm{AB}$ \\
glyphosate $+2,4-\mathrm{D}(0,65+0,81)$ & 5,6 & $\mathrm{BC}$ \\
glyphosate $(1,30)$ & 4,9 & $\mathrm{C}$ \\
glyphosate $(0,65)$ & 2,9 & $\mathrm{C}$ \\
glyphosate $+2,4-\mathrm{D}(0,97+1,22)$ & 2,9 & $\mathrm{C}$ \\
glyphosate $+2,4-\mathrm{D}(0,81+1,01)$ & 2,1 & $\mathrm{C}$ \\
glyphosate $+2,4-\mathrm{D}(1,30+1,62)$ & 1,3 & $\mathrm{C}$ \\
glyphosate $+2,4-\mathrm{D}(1,13+1,42)$ & 1,0 & $\mathrm{C}$ \\
\hline F trat & 14,10 & $* *$ \\
C.V.(\%) & 43,5 & \\
D.M.S. (Tukey) a 5\% & 6,0 & \\
\hline
\end{tabular}

(1) Dosagens em kg i.a./ha

(2) Dados transformados em $\sqrt{X+1}$

D.A.A. - Dias Após a Aplicação

\section{AGRADECIMENTOS}

Os autores agradecem à MONSANTO DO BRASIL - DIVISÃO AGRICULTURA, pela cessão de parte dos herbicidas utilizados nos ens aios, bem como pelo empenho no auxílio à busca de bibliografias sobre os as suntos estudados, tão escassas nas bibliotecas brasileiras.

\section{LITERATURA CITADA}

ALAM (Asóciacion Latinoamericana de Malezas). Recomendaciones sobre unificación de los sistemas de avaluacion en ensayos de control de malezas. ALAM, Bogotá, v.1, n.1, p.35-38, 1974.

CARVALHO, J.E.B.; REZENDE, G.O.; PEIXOTO, C.P.; PINHO, A.F.S.; FOLONI, L.L. Avaliação da eficiência do produto 2,4-D + glifosate no controle de plantas daninhas na cultura do cacau. In: CONGRESSO BRASILEIRO DE HERBICIDAS E PLANTAS DANINHAS, 18, 1991 a, Brasilia. Resumos, Brasília: SBHED, 1991 a, p.95-96. 
CARVALHO, J.E.B.; REZENDE, G.O.: FOLONI, L.L.; MOTA, P.P. Estudo da efi ciência da mistura pronta de 2,4-D + glyphosate no controle de plantas daninhas em citros. In: CONGRESSO BRASILEIRO DE HERBICIDAS E PLANTAS DANINHAS, 18, 1991 b, Brasilia. Resumos, Brasília: SBHED, 1991 b, p.103-104.

CRUZ, L.S.P.; PIRES, E.J.P.; FAHL, J.I. Efeitos de misturas prontas com diuron e paraquat no controle de plantas daninhas em citrus (Citrus sinensis Osbeck. Natal) em produção. In: CONGRESSO BRASILEIRO DE FRUTICULTURA, 8, v.1, Brasília, 1986. Anais. p.231-236.

DE NEGRI, J.D. Práticas culturais para aumento da produtividade dos citros. In: Ill SIMPÓSIO DE CITRICULTURA, Jaboticabal, São Paulo, 1988. Anais. p.205-219.

DURIGAN, J.C. Controle químico de plantas daninhas na citricultura. Jaboticabal, FUNEP/FCAV-UNESP, 1988. 18 p.

DURIGAN, J.C.; GALLI; A.J.B .; LEITE，G.J. Avaliação da eficiência da mistura de glyphosate e 2,4-D para o controle de plantas daninhas em citros. In: CONGRESSO BRASILEIRO DE HERBICIDAS E PLANTAS DANINHAS, 17, 1988, Piracicaba. Resu mos, Piracicaba: SBEHD, 1988, p.303304.

EWRC (European Weed Research Council). Report of 3rd and 4th meetings of EWRC Committee of Methods in Weed Research. Weed Res., v.4, n.1, p.88, 1964.

FLINT, J.L.; BARRET, M. Effects of glyphosate combinations with 2,4-D or dicamba on field bindweed (Convolvulus arvensis). Weed Sci., v.37, n.1, p.12-18, 1989.
GALL I, A.J.B. Avaliação da ef ic iência de glyphosate em mistura com diversos produtos, no controle de Commelina virginica (trapoeraba) em citros. In: CONGRESSO BRASILEIRO DE HERBICIDAS E PLANTAS DANINHAS, 18, 1991, Brasilia. Resumos, Brasília: SBHED, 1991, p.104-105.

IBGE. Fundação Instituto Brasileiro de Geografia e Estatística. Anu. Estat.. v.52, p.1-1119, 1992.

HAMMERTON, J.L. Weed problems and weed control in the Commonwealth Caribbean, Trop. Pest. Manage.. v.27, n.3, p.379-387, 1981.

LANGE, A.; DAY, B.; PHILLIPS, R,; TUCKER, D.; KLOSTERBOER, A. Weed control in citrus. In: Citrus. Bulletin Ciba-Geigy, 1975. p.55-60.

LORENZI, H. Plantas daninhas do Brasil: terrestres, aquáticas, parasitas, tóxicas e medicinais. Nova Odessa, 1982. 425p.

LORENZI, H. Manual de identificação e controle de plantas daninhas: plantio dir eto e con ven cional. 2" ed. Nova Ode ssa, 1986. 240p.

MOSHIER, L.J. Response of honeyvine milkweed (Ampelamus alhidus) to herbicides aplications. Weed Sci.. v.28. p.722-724, 1980.

SUWUNNAMEK, U.; PARKER, C. Control of Cyperus rotundus with glyphosate: the influence of ammonium sulphate and others additives. Weed Res., v.15, p.13-19, 1975.

TOLLERVEY, F.E.; FRANS, R.; PANIAGUA, O.; LARA, R. Weed control investigations in Bolivi an crops 1977-1978. Weed Mist., v.29, n.7, p.225, 1979. 